\title{
SIMULATION OF THE SPECTRAL CHARACTERISTICS OF THE PHOTOSENSITIVE ELEMENTS BASED ON THE POLYCRYSTALLINE LEAD SULFIDE
}

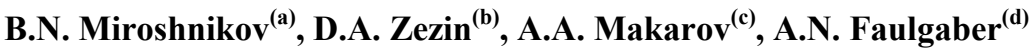 \\ (a),(b),(c),(d) National Research University “Moscow Power Engineering Institute", Moscow, Russia

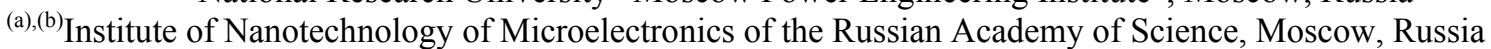 \\ ${ }^{(c)}$ International Laboratory of Statistics of Stochastic Processes and Quantitative Finance of the National Research Tomsk \\ State University, Tomsk, Russia \\ (a) MiroshnikovBN@mpei.ru, ${ }^{(b)}$ ZezinDA@mpei.ru, ${ }^{\left({ }^{(c)}\right.}$ o_v_ch@mail.ru, ${ }^{(\mathrm{d})}$ lytaleksandr@mail.ru
}

\begin{abstract}
The results are presented of the simulation of the spectral characteristics of the polycrystalline photosensitive elements based on the lead sulphide. The possible reasons for the experimental data discrepancy are discussed.
\end{abstract}

Keywords: photoresistors, lead sulfide, carrier lifetime, photosensitivity, spectral dependence

\section{INTRODUCTION}

Lead sulfide $(\mathrm{PbS})$ is one of the most needed narrowband-gap semiconductors with the energy-gap width of $0.4 \mathrm{eV}$ at $300 \mathrm{~K}$. The photoresistors based on it are used in registering near infrared radiation (0.6-3.1 microns), i.e. they are integrated into impulse optoelectronic devices, IR detectors, infrared imagers, night viewing devices, etc.

A wide variation of the methods for obtaining the devices mentioned above leads to the production of the patterns of various thicknesses, surface morphology and structure with the result that the spectral characteristics of the devices vary widely.

The objective of this paper is to show the possibility of simulation of these photosensitive elements and to discuss the likely reasons why their theoretical characteristics differ from the experimental curves.

\section{SIMULATION OF THE SPECTRAL CHARACTERISTICS OF PHOTORESISTORS}

As a basis, the experimental data presented by Ravich, Efimova and Smirnov (1968) are used. Mobility is estimated as $1 \mathrm{~cm}^{2} / \mathrm{V} \cdot \mathrm{s}$; carrier (hole) lifetimes $\tau$ are chosen to be $50 \mu \mathrm{s}$ for the "physical" element and 100 and $300 \mu$ s for the "chemical" element. The geometrical dimensions are: the photosensitive element area is $1 \mathrm{~cm} \times 1 \mathrm{~cm}$, the thickness is 0.5 micron for the chemical layer and 1 micron for the physical layer. As a radiation source, the absolute black body is used. The temperature of the black body is $500 \mathrm{~K}$, while its radiation spectrum is determined based on the Planck radiation formula. The relationship between the absorption coefficient and the incident radiation energy is taken from the study by Miroshnikov, Miroshnikova and Popov (2018).

In the general case, the dark carrier concentration is determined by the two components. They are: $p_{1}$ related to the Auger recombination and carrier lifetime and obeying the ratio $\tau \cdot p_{1}^{2}=(2 \ldots 3) \cdot 10^{27} \mathrm{~cm}^{-6} \cdot \mathrm{s}$; and $p_{2}$ determined by the concentration of the acceptortype traps and, especially, by the concentration of both chemically bound oxygen (it forms the deeper traps and increases the surface recombination rate at the crystallite surface) and physically adsorbed oxygen at the crystallite surface (Fig. 1). Our studies (Mohamed, Abdel-Hafiez, Miroshnikov, Barinov and Miroshnikova 2014) show that by heating the patterns up to the temperature of $400 \mathrm{~K}$ and degassing the surface, one can minimize the last component and obtain the minimum hole concentration which depends on the shallow trap concentration and is related to the carrier lifetime by the law

$p_{2}=1.4 \cdot 10^{20} \tau$

Thus, the dark hole concentration

$p_{\text {dark }}=p_{\text {dark }}(\tau)=p_{1}+p_{2}$

depends on the carrier lifetime. For example, in Fig. 1, there are presented the dependences $p_{1}, p_{2}$ (1) and $p_{\text {dark }}(2)$ as the functions of the carrier lifetime for the lead chalcogenides.

The carrier concentration in the presence of light $\left(p_{\text {gen }}\right)$ is determined by the generation rate $G(\lambda, x)$ depending on both the radiation wavelength $\lambda$ and the photon penetration depth $x$ :

$G(\lambda, x)=\alpha(\lambda) N_{p}\left(\lambda, T_{\mathrm{ABB}}\right) \eta(\lambda) \mathrm{d} x$, 


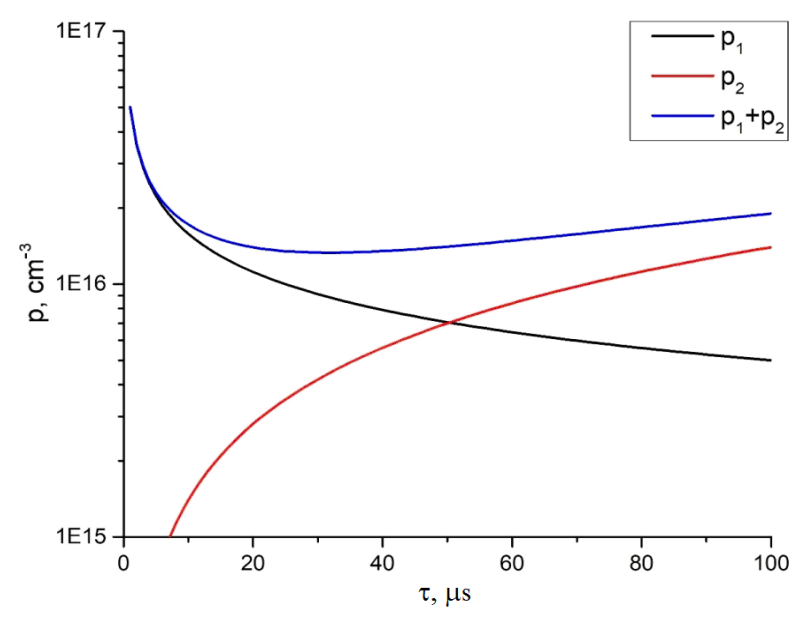

Figure 1: The dependences of the hole concentration in lead chalcogenides upon the carrier lifetime

where $\alpha(\lambda)$ is the radiation absorption coefficient; $N_{p}\left(\lambda, T_{\mathrm{ABB}}\right)$ is the number of photons incident in a unit of time (second) per unit of area $\left(\mathrm{cm}^{2}\right)$ and depending on both the radiation wavelength $\lambda$ and the temperature of the radiation source (absolute black body - ABB); $\eta(\lambda)$ is the quantum efficiency of the photoresistor close to unity. Thus, the total carrier concentration $p_{l t}$ is determined by the sum of the dark carrier concentration $p_{\text {dark }}(2)$ and the carrier concentration $p_{\text {gen }}$ under the lighting by source (ABB) radiation:

$p_{l t}(\tau, \lambda)=p_{\text {dark }}(\tau)+p_{\text {gen }}(\tau, \lambda)$

And, in turn, $p_{\text {gen }}(\tau, \lambda)=G(\lambda, x) \tau$.

In order to find the spectral dependence $S_{I}(\lambda)$ of the current sensitivity, the following formula is applied:

$S_{I}(\lambda)=I_{p}(\lambda) / F\left(\lambda, T_{\mathrm{ABB}}\right)$

In Eq. (4), the designations are: $I_{p}(\lambda)$ is the photocurrent, $F=N_{p} h v=N_{p} h c / \lambda$ is the photon flux, $N_{p}$ is the number of photons, $h$ is the Planck constant, $c$ is the velocity of light in free space, $v$ is the electromagnetic radiation frequency. For the photocurrent $I_{p}(\lambda)$, one has:

$$
I_{p}(\lambda)=V_{d} / R_{l t}(\lambda)
$$

where $V_{d}$ is the bias voltage (which is equal to $15 \mathrm{~V}$ ) and

$$
R_{l t}(\lambda)=\rho_{l t}(\lambda) a / b d=\rho_{l t}(\lambda) / d=1 / \sigma_{l t}(\lambda) d
$$

is the incident radiation photoresistor resistance. Here $a \times b$ is the photosensitive element effective area which has been taken as square and equal to $1 \mathrm{~cm}^{2}$ for the calculations; $d$ is the photosensitive layer thickness; $\rho_{l t}(\lambda)$ is the light photosensitive element resistivity; $\sigma_{l t}(\tau, \lambda)$ is the light photosensitive element conductivity.

The conductivity $\sigma_{l t}(\tau, \lambda)$ is primarily determined by the conductivity $\sigma_{p}(\tau, \lambda)$ due to the majority carriers, that is, holes:

$\sigma_{l t}(\tau, \lambda)=\sigma_{n}(\tau, \lambda)+\sigma_{p}(\tau, \lambda) \approx \sigma_{p}(\tau, \lambda)=q p_{l t}(\tau, \lambda) \mu_{p}$

where $q$ is an elementary charge, $\mu_{p}$ is the hole mobility close to $1 \mathrm{~cm}^{2} / \mathrm{V} \cdot \mathrm{s}$ and independent from the light level in the first approximation, while $p_{l t}(\tau, \lambda)$ is determined by the formula (3).

There are two main moses of absorption: near-surface (or non-uniform) absorption and uniform absorption. From the Bouguer-Lambert law, it follows that all the absorption of the radiation incident on the surface occurs at the depth $x=\alpha^{-1}(\lambda)$. Then, when the ratio $\alpha(\lambda) d>1$ is fulfilled, it was accepted that all the incident radiation is absorbed at a depth less than the film thickness, i.e. on the surface. On the other hand, if the inequality $\alpha(\lambda) d<1$ is satisfied, then it was assumed that the absorption is uniform.

In the simulation of the lead sulfide thin layers obtained by chemical deposition, it was presupposed that all the absorption is uniform. For thicker physical layers, both types of absorption were taken into account. In addition, the difference in the film technology also influences the efficiency of the radiation absorption by the layers $\eta(\lambda)$. Thus, the chemical layers are characterized by a large energy-gap width, and, therefore, radiation wavelength longer than 3 microns is not absorbed, while for the physical layers, absorption in the nearsurface region has less influence due to the thick layer of lanarkite $\left(\mathrm{PbO} \cdot \mathrm{PbSO}_{4}\right)$.

For our low-mobility samples, the condition $L_{p}<<d$ is satisfied, that is, the hole diffuse length $L_{p}$ is much less than the photosensitive element thickness $d$, and there can be applied the formula for the concentration under the uniform absorption:

$p_{\text {gen }}(\tau, \lambda)=G(\lambda, x) \tau\left[1-\frac{2 s L_{p} \tau}{d\left(L_{p}+s \tau\right)}\right] \approx G(\lambda, x) \tau$.

where $s$ is surface recombination rate.

For the near-surface absorption, one gets:

$p_{\text {gen }}(\tau, \lambda)=G(\lambda, x) \tau \frac{L_{p}}{\alpha(\lambda) d\left(L_{p}+\tau s\right)}\left[\frac{\tau s}{L_{p}\left(\alpha(\lambda) L_{p}+1\right)}+1\right]$. 


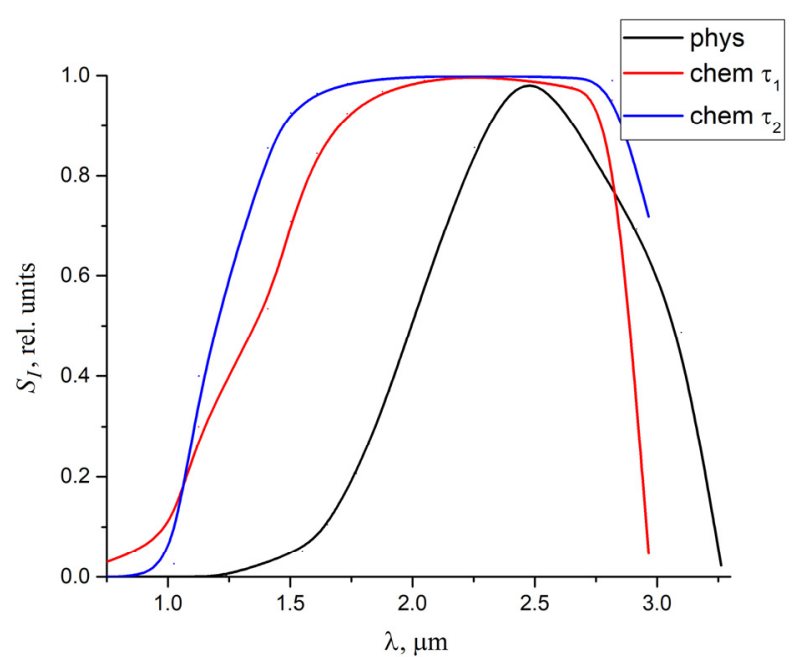

Figure 2: The calculated spectral characteristics for the photosensitive layers with the three lifetimes

In Fig. 2, there are presented the three calculated spectral characteristics of the photosensitive element: phys - for the physically deposited layer with $\tau=50$ $\mu \mathrm{s}$, chem $\tau_{1}$ - for the chemically deposited layer with $\tau=100 \mu$ s and chem $\tau_{2}$ - for the chemically deposited layer with $\tau=300 \mu \mathrm{s}$.

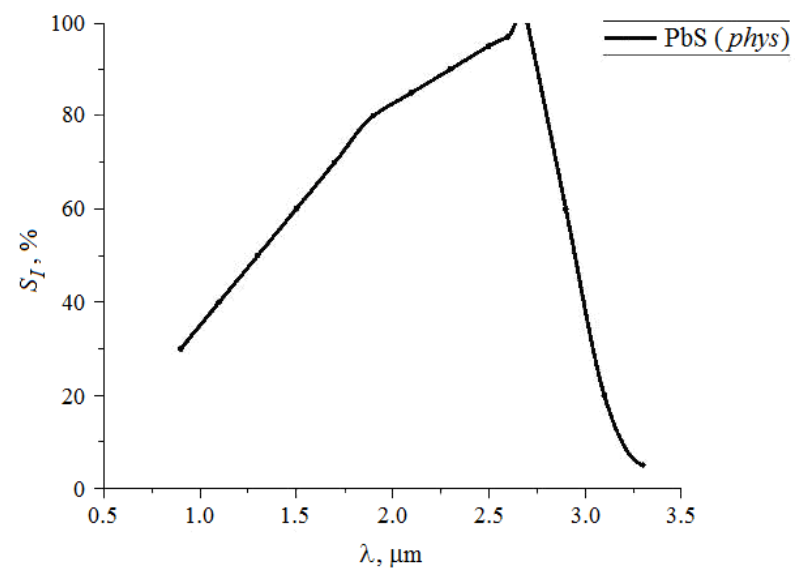

a)

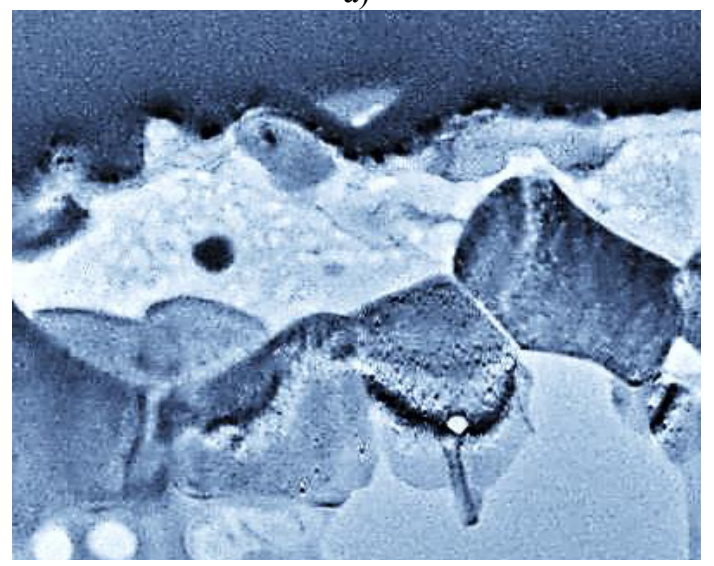

b)

Figure 3: The experimental spectral characteristics (a) and the microphotography of the physical photosensitive element section obtained by means of the transmission high-resolution electron microscope (b)
Our calculations and the comparison with the experimental data show that, for the physical layer, the transition from the near-surface absorption to the uniform one is realized when the value of the incident radiation wavelength is no less than 2 microns (curve phys in Fig. 2). If the incident radiation wavelength is bigger than 2.7 microns, then the absorption efficiency near the energies equal to the energy-gap width begins to be influential and the sensitivity begins decreasing. In turn, for the chemical layers (curves chem in Fig. 2), the uniform absorption is only taken into account, therefore, in the large wavelength range from 1.5 microns to 2.7 microns, the typical constant high reduced sensitivity is observed. The difference has also been determined under the various values of the lifetime affecting both the dark hole concentration $p_{\text {dark }}(\tau)$ and the generated carrier concentration $p_{\text {gen }}(\tau, \lambda)$.

\section{DISCUSSION OF THE RESULTS}

For the physically deposited layer, the simulation coincides well with the experimental data (Fig. 3, Morozova and Miroshnikov 2018).

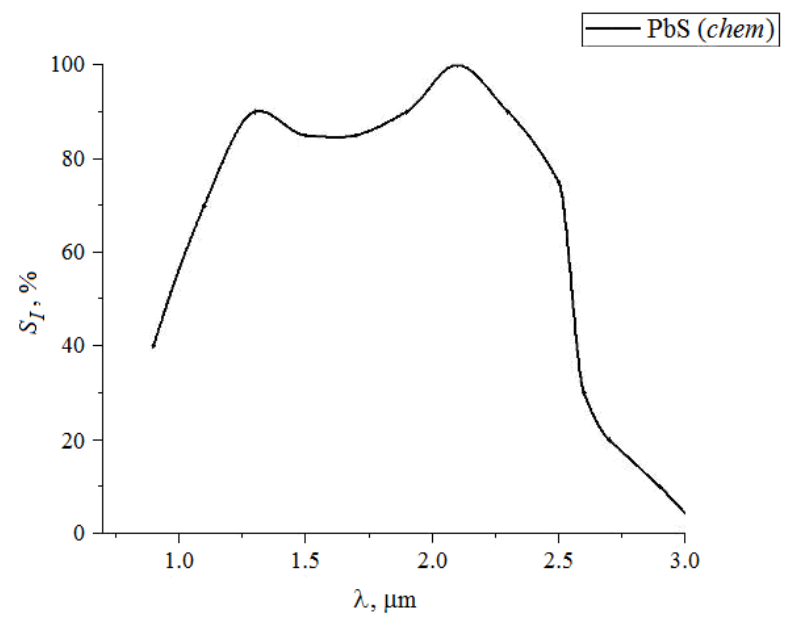

a)

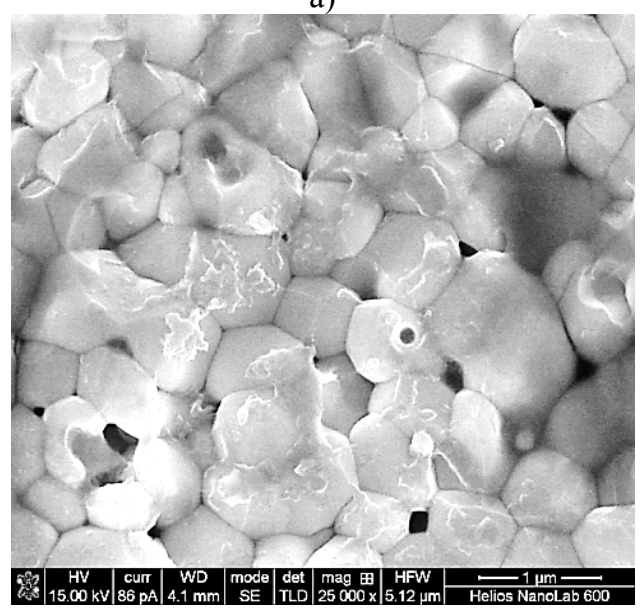

b)

Figure 4: The experimental spectral characteristics (a) and the microphotography of the section of the photosensitive element surface obtained by means of the chemical deposition with the maximum sensitivity (b) 
The difference in the slope of the curve in the highenergy spectral region (up to 2.3 microns) is determined by the thickness of the non-photoactive layer of the lead oxides colored by the light colors in Fig. $3 \mathrm{~b}$.

The results of the simulation of the spectral characteristics of the photosensitive element obtained by the chemical method also agree well with the experimental data (Fig. 4, Morozova and Miroshnikov 2018). What has not been taken into account in this case is the change in the lead sulfide energy-gap width due to the effect of the oxygen in $\mathrm{PbS}$ structure that is the isoenergetic impurity resulting in a local change in the energy-gap width of the material (Morozova and Miroshnikov 2018). This is the first reason for the conformity between the simulation results and the experimental data. Another reason is the small photosensitive element thickness -0.5 micron, resulting in the long-wavelength (low-energy) radiation part passing through the film without the photo-ionization.

\section{CONCLUSION}

Thus, it has been established that the simulation can be implemented of such complex structure as the polycrystalline photosensitive element and its results appear to be close to the experimental data.

The simulation takes into account relaxation processes both inside the crystallites (Auger recombination) and the effect of oxygen-containing impurities on the surface, leading to the capture of free electrons, an increase in the hole lifetime and stabilization of the dark conductivity.

\section{ACKNOWLEDGMENTS}

This research was financially supported by the Russian Foundation for Basic Research (research project No. 1829-11051), the Ministry of Education and Science of the Russian Federation (research project No. 2.3208.2017/4.6), and the Council on grants of the President of the Russian Federation (research project No. SP-834.2019.3).

\section{REFERENCES}

Ravich Y.I., Efimova B.A. and Smirnov I.A., 1968. Methods for the study of semiconductors as applied to $\mathrm{PbTe}, \mathrm{PbSe}, \mathrm{PbS}$ lead chalcogenides [in Russian]. Moscow: Nauka.

Miroshnikov B.N., Miroshnikova I.N. and Popov A.I., 2018. Optimization of parameters of PbS-based polycrystalline photoresistors. Semiconductors or Physics of the Solid State, 52 (2), 245-249.

Mohamed H.S.H., Abdel-Hafiez M., Miroshnikov B.N., Barinov A.D. and Miroshnikova I.N., 2014. Spectral characteristics and morphology of nanostructured $\mathrm{Pb}-\mathrm{S}-\mathrm{O}$ thin films synthesized via two different methods. Journal of Materials Science in Semiconductor Processing, 27, 725732 .

Morozova N.K. and Miroshnikov B.N., 2018. Isoelectronic oxygen centers and conductivity of
$\mathrm{CdS}$ in comparison with $\mathrm{PbS}$. Semiconductors or Physics of the Solid State, 52 (3), 295-298.

\section{AUTHORS BIOGRAPHY}

Boris N. Miroshnikov was born in Moscow in 1990. He received his Ph.D. in semiconductor physics from the National Research University "Moscow Power Engineering Institute" in 2016. Boris N. Miroshnikov is currently working as an Assistant Professor at the Department of Electronics and Nanoelectronics of the National Research University "Moscow Power Engineering Institute". He also works as a Researcher at the Institute of Nanotechnologies of Microelectronics of the Russian Academy of Science. His main interests are physics and technology of infrared photodetectors based on single- and polycrystals.

Denis A. Zezin was born in 1988. He received his $\mathrm{Ph} . \mathrm{D}$. in solid-state electronics from the National Research University "Moscow Power Engineering Institute" in 2014. He is currently working as an Associate Professor at the Department of Electronics and Nanoelectronics of the National Research University "Moscow Power Engineering Institute" as well as a Researcher at the Institute of Nanotechnologies of Microelectronics of the Russian Academy of Science. His research interests relate to the study of the electrophysical visible and infrared radiation-measuring instruments.

Alexander A. Makarov was born in 1993. He received the B.Sc. and the M.Sc. degrees from the National Research University "Moscow Power Engineering Institute" in 2014 and 2016, respectively, both in radio engineering. Presently, Alexander A. Makarov is a PhD student in the Department of Electronics and Nanoelectronics at the National Research University "Moscow Power Engineering Institute" as well as he is working as a Researcher at the International Laboratory of Statistics of Stochastic Processes and Quantitative Finance of the National Research Tomsk State University. His research interests include synthesis, analysis and simulation of the processing algorithms of stochastic processes and fields with unknown parameters.

Alexander N. Faulgaber was born in 1993. He received the B.Sc. and the M.Sc. degrees from the National Research University "Moscow Power Engineering Institute" in 2014 and 2016, respectively, both in radio engineering. Presently, Alexander N. Faulgaber is a $\mathrm{PhD}$ student in the Department of Electronics and Nanoelectronics at the National Research University "Moscow Power Engineering Institute". His research interests include synthesis, analysis and simulation of the processing algorithms of stochastic processes and fields with unknown parameters. 